\title{
Aplicaciones CAE en el diseño de moldes de inyección de termoplásticos 1
}

Jorge Fajardo Seminario John Calle Sigüencia Ingeniería Mecánica UPS-CUENCA

El grupo de materiales que más ha crecido en su aplicación es sin duda el de los polímeros;, que han alcanzado volúmenes de aplicación como materia prima sobre el $80 \%$ con extensas aplicaciones en todas las ramas industriales.

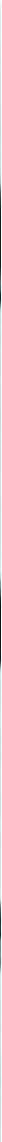




\section{Antecedentes}

En la actualidad la gran mayoría de los procesos de producción en serie se fundamentan en el diseño de nuevos productos, en la ingeniería de los materiales y en la ingeniería de los procesos, por ello se han creado productos que requieren la utilización de materiales con mejores propiedades y cuyos procesos de transformación garantizan la calidad de los productos sin reducir la eficiencia productiva y económica. El grupo de materiales que más ha crecido en su aplicación es sin duda el de los polímeros; que han alcanzado volúmenes de aplicación como materia prima sobre el $80 \%$ con extensas aplicaciones en todas las ramas industriales.
A partir de las herramientas $C A E$, el diseño de las piezas plásticas se estudia desde el punto de vista mecánico-resistente y desde el punto de vista de la fabricabilidad de las mismas, mediante la tecnología de inyección.

\section{Uentajas}

Las tecnologías modernas de diseño, análisis, manufactura y generación de prototipos rápidos (CAD/CAM/CAE y Rapid Prototiping) son herramientas indispensables para la empresa manufacturera actual. Ya que posibilitan:

- Acortar el Ciclo de Diseño-Manufactura.
- Evaluar Alternativas de Diseño y Simular los Procesos de Fabricación.

- Evitar Costosos Errores durante el Proceso de Manufacturación.

- Elevar la Calidad de los Productos y de la Información Técnica.

- Diseñar, Analizar, Fabricar y Vender sin Perder el Tiempo por Ensayo y Error.

\section{Programas}

Los paquetes que se pueden encontrar en la actualidad con aplicación directa en el diseño y producción de piezas plásticas son entre otros:

\begin{tabular}{|l|l|l|}
\hline \multicolumn{1}{|c|}{ PROCESO } & \multicolumn{1}{|c|}{ SOFTWARE } & \\
\hline Inyección & Mold Flow & $\begin{array}{l}\text { Modiflow Plastic Insight (MPI) } \\
\text { Modiflow Plastic Adviser (MPA) }\end{array}$ \\
\hline Extrusión & Flow 2000 & \\
\hline Soplado & B-Sim & \\
\hline Termoconformado & T-Sim & \\
\hline \hline
\end{tabular}

\section{Aplicación universitaria y productiva}

En la Universidad Politécnica Salesiana, se han venido incorporando estas aplicaciones CAE dentro de las diversas áreas de diseño, mediante proyectos de tesis en los que se han generado aplicaciones concretas que han servido para validar los resultados obtenidos por medio de la simulación, como el que se trata en este artículo, específicamente del programa MPI 3.1.
Los resultados que se han obtenido por medio de cálculos analíticos empleando métodos de resolución numérica, aplicación de diagramas y mediante la simulación en MPI 3.1 han mostrado gran similitud, con diferencias porcentuales que van desde el $0.25 \%$ al $20 \%$, lo que valida indiscutiblemente la fiabilidad de estos resultados.

\section{moldflow}

La familia Modlflow Plastic Insight (MPI) posee módulos con capacidad de simular el llenado, empaquetamiento y enfriamiento del molde (multicavidad y/o familiar). Los dos primeros módulos son para simular el llenado y empaquetamiento del molde (MPI/Fusion y MPI/Flow), en este caso no solamente se introducen los parámetros mencionados anteriormente, sino que también se introduce la máquina con la cual se va inyectar el molde, la relación d/l, el recorrido del pistón, la velocidad, cómo y cuándo se va a hacer el cambio de presión de inyección a presión sostenida y cuál debe ser este tiempo. 


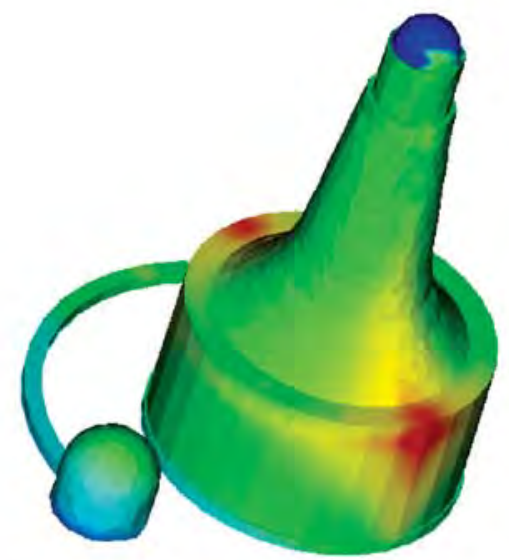

notoftion

Figura 1.- Determinación del mejor punto de inyección mediante programa MPI.3.1

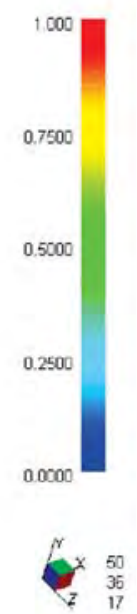

A partir de las herramientas CAE, el diseño de las piezas plásticas se estudia desde el punto de vista mecánico-resistente y desde el punto de vista de la fabricabilidad de las mismas, mediante la tecnología de inyección.

En la Figura 1 se puede observar una aplicación del módulo de llenado para determinar el punto más adecuado para realizar la inyección de una tapa con capuchón en Polietileno de alta densidad.

En la Figura 2 se despliega la animación del resultado de cálculo de la presión específica que ejerce el material plástico sobre la cavidad del molde.

Las tecnologías modernas de diseño, análisis, manufactura y generación de prototipos rápidos [CAD/CAm/CAE y Rapid Prototiping] son herramientas indispensables para la empresa manufacturera actual.
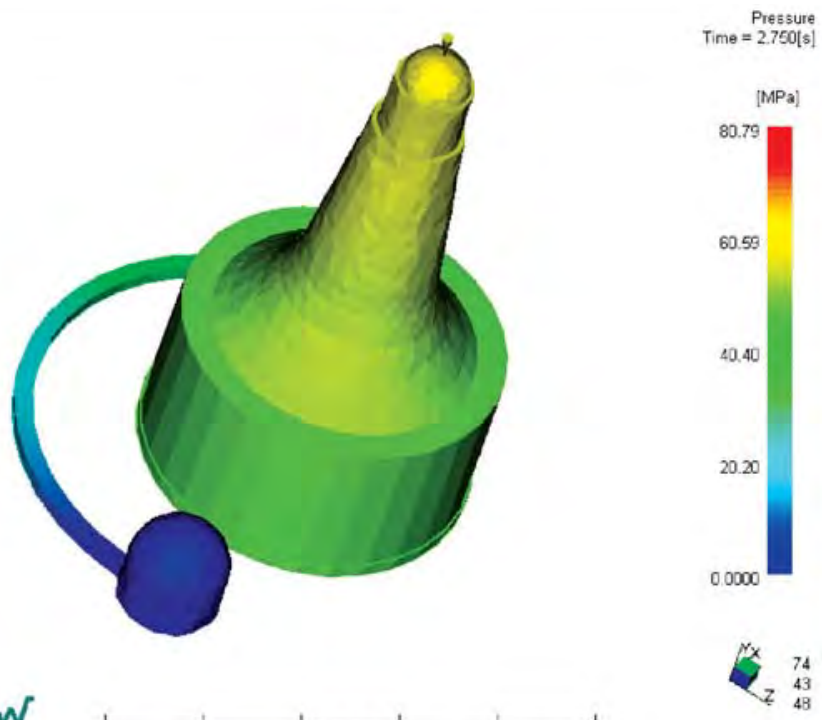

Notoftow

Scale (50 mm)

Figura2.- Cálculo de la presión de inyección mediante programa MPI.3.1

De igual manera se puede visualizar las zonas en donde tendrá lugar los atrapamientos de aire al interior de la pieza inyectada.

A partir de estos parámetros de diseño se pueden efectuar las correcciones necesarias a fin de garantizar un producto final con las exigencias de calidad requeridas. 

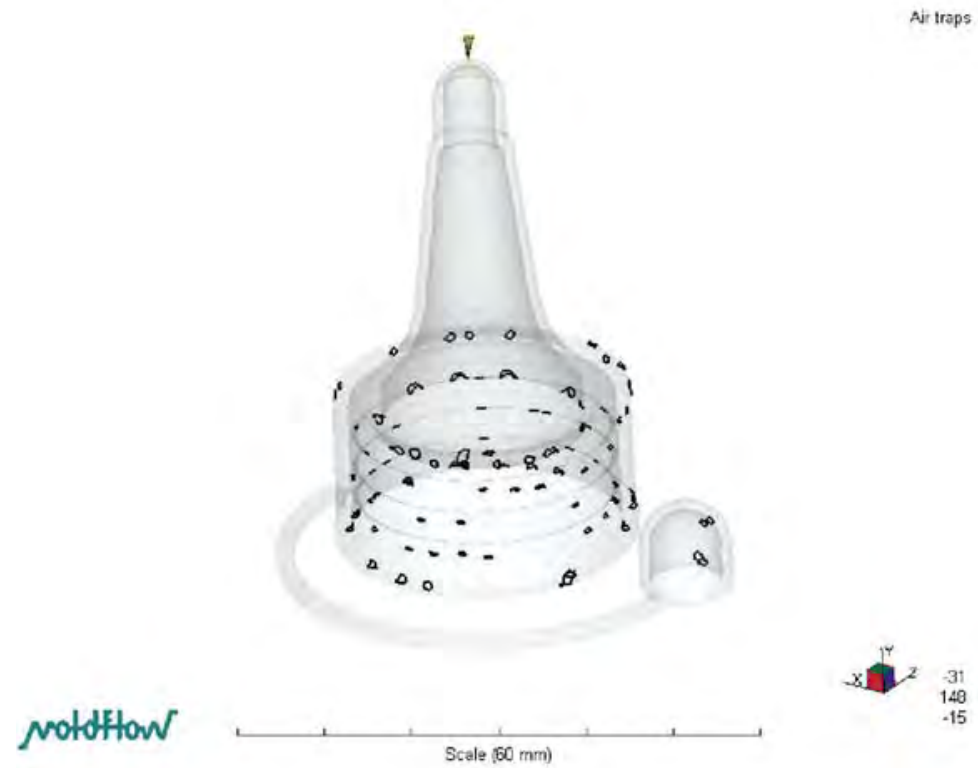

Determinación de los puntos de atrape de aire mediante

programa MPI.3.1

El tercer módulo es el MPI/Cool, con éste se simula la etapa del enfriamiento, para eso cuenta con una base de datos de los metales más utilizados para hacer moldes, así como una base de datos de los refrigerantes más comunes, este módulo permite al usuario dibujar el sistema de refrigeración, y darle los parámetros necesarios para obtener resultados como: la distribución final de la temperatura de la pieza, la temperatura de salida del refrigerante, si debe o no llevar insertos de otro material (CuBe), si debe o no llevar baffles o bublers, No. de Reynolds, distribución de la temperatura en toda la superficie del molde, etc. A partir de este módulo, existen múltiples módulos muy específicos dependiendo del tipo de inyección que se realiza o el resultado final que se quiere estudiar, alguno de ellos son: MPI/Warp (Alabeo y deformaciones), MPI/Stress (estudio de cargas), MPI/Gas (Inyección asistida por gas), MPI/RIM (Inyección de hule y/o termoestables), etc.
En la figura se muestra un reporte de la variación de temperatura que alcanza el líquido de refrigeración al interior de los canales, producto de la transferencia de calor en la etapa de atemperado de la pieza inyectada.

Es posible determinar las deformaciones y contracciones que se producirán en las piezas una vez que han sido moldeadas, en el siguiente reporte se pone de manifiesto el efecto de las contracciones en todas las direcciones:

\section{Es vital encontrar parámetros propios de diseño, enmarcados en las normas correspondientes con aplicación de elementos estandarizados empleando mo- delos matemáticos...}

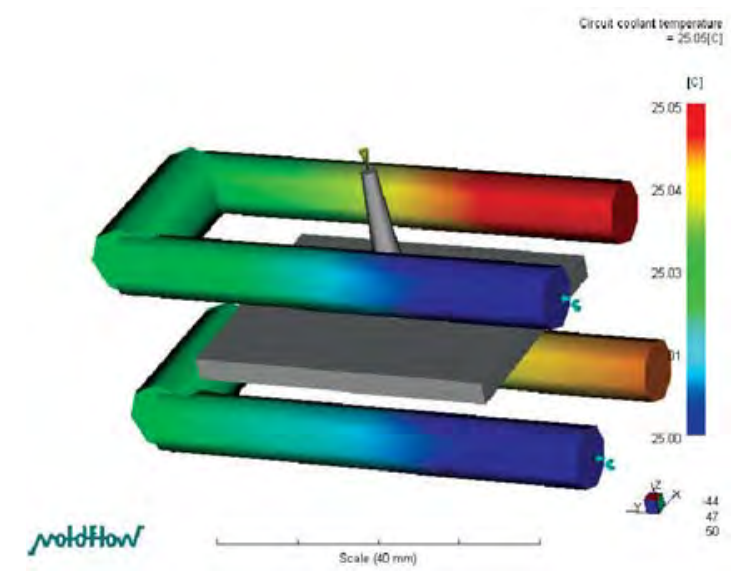

Determinación de la temperatura del líquido refrigerante mediante programa MPI.3.1 


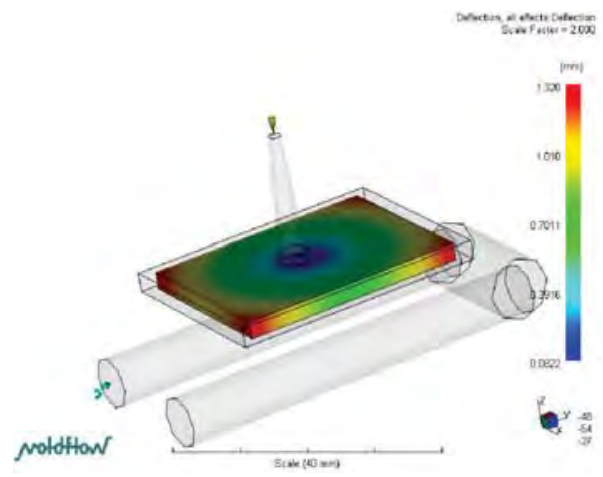

Determinación de la contracción volumétrica mediante programa MPI.3.1

El archivo de entrada para cualquiera de estos programas es un archivo de extensión. STL, si el CAD elegido genera este archivo no existe ningún problema, los CAD más reconocidos son: Pro-E, Catia, Inventor o Mechanical Desktop y Solidworks.

\section{Innouando}

Es vital encontrar parámetros propios de diseño, enmarcados en las normas correspondientes con aplicación de elementos estandarizados empleando modelos matemáticos y con la gran ayuda de sistemas de simulación por computador que brinden seguridad en los procesos.

La recomendación para utilizar cualquiera de estos programas depende más que todo del número de moldes a fabricar por año, así como del tipo de piezas, y los problemas más comunes que se tienen con los re-trabajos de los moldes. Una vez que la pieza pasa las pruebas de manufacturabilidad y tiene la aprobación del departamento de diseño de producto, entonces es cuando en el departamento del taller de moldes se empieza a diseñar por completo todo el molde y para esta etapa lo más recomendable es utilizar el MPI, el usuario de este programa debe conocer en detalle cuales son las características más importantes del diseño de moldes, así como estar en la capacidad de ajustar parámetros de inyección en la simulación.

Concientes de la importancia que tiene el diseño dentro de la Ingeniería Mecánica, la Universidad Politécnica Salesiana sigue incursionando en el conocimiento, generación y aplicación de estas herramientas que vuelven a la práctica del diseño en una tarea de mayor eficiencia dentro del campo de la producción industrial.

\section{La recomendación para utilizar cualquiera de estos programas depende más que todo del número de moldes a fabricar por año, así como del tipo de pie- zas, y los problemas más comunes que se tienen con los re-trabajos de los moldes.}

1 Información proporcionada por ATGroup Software, representantes exclusivos de Moldflow en Latinoamérica.

Reportes gráficos tomados de Tesis De Ingeniería "Optimización del proceso de diseño de moldes para termoplásticos". Jorge Fajardo, S. Ingeniería Mecánica. 